\title{
Glossary of notation
}

- Data and metadata

- A: $i \times j$ binary matrix of microbial presence-absence, where $i$ is the number of microbes, $j$ is the number of samples, and $a_{m, n}$ is 1 when microbe $m$ is observed in sample $n$ and 0 otherwise

- $a_{m, N}$ : a vector of presence-absences for microbe $m$ in samples $n \in N$

$-e_{x}, d_{y}$ : environment $x$ or study population $y$, each corresponding to a set of samples

$-E=\left\{e_{1}, \ldots, e_{k}\right\}$ : the set of environments being studied or compared (e.g., body sites; health vs. disease)

$-D=\left\{d_{1}, \ldots, d_{l}\right\}:$ the set of study populations

- Linear models

$-\vec{\phi}_{x, E[, D]}(A)$ : phenotype vector, calculated for environment $e_{x} \in E$ and potentially adjusting for dataset effects $D$, based on microbial presence-absence matrix $A$, with elements corresponding to phenotype estimates for individual microbes

- $\vec{\phi}_{x, E, D}^{\text {Prev }}(A)$ : prevalence phenotype estimates (based on $\operatorname{logit}\left(\hat{p}_{m, N, D}^{\mathrm{ADDW}}(A)\right)$; see below).

$-\vec{\phi}_{x, E}^{\mathrm{Spec}}(A)$ : environmental specificity score phenotype estimates (based on $\operatorname{logit}\left(\widehat{s}_{m, x, E}^{\mathrm{MAP}}(A ; b)\right) ; \operatorname{see}$ below).

$-\beta_{0, g}$ : in the linear model for gene $g$, intercept term used to model the average value of a given phenotype $\vec{\phi}_{x, E}(A)$

$-\beta_{1, g}$ : in the linear model for gene $g$, the effect of having vs. not having gene $g$ on a given phenotype $\vec{\phi}_{x, E}(A)$

$-\vec{I}_{g}$ : the binary vector of gene presence-absence whose elements are $I_{g, m}$, equal to 0 if the gene $g$ is absent in microbe $m$ and 1 if it is present

- Phenotype estimation

- $p_{m, N}$ : prevalence, the probability of observing a microbe $m$ in a set of samples $N P(m \mid N)$

- $\hat{p}_{m, N}^{\mathrm{MLE}}(A)$ : the maximum-likelihood estimate of prevalence, based on the presence-absence matrix A

- $\hat{p}_{m, N}^{\mathrm{ADD}}(A)$ : an estimate of prevalence based on the presence-absence matrix $A$ using additive smoothing

- $\hat{p}_{m, N, D}^{\mathrm{ADDW}}(A)$ : an estimate of prevalence based on the presence-absence matrix $A$ using additive smoothing, and additionally weighting by the inverse number of samples per dataset in $D$

- $\hat{p}_{m, E}^{\mathrm{EnvW}}(A)$ : an estimate of the prevalence across environments, weighted by their probability (i.e., $P(m)$ obtained by marginalizing $\left.P\left(m \mid e_{x}\right)\right)$

$-s_{m, x, E}$ : environment specificity, the probability of being in a particular environment $e_{x}$ given that microbe $m$ was observed $P\left(e_{x} \mid m\right)$

$-\hat{s}_{m, x, E}(A)$ : an estimate of environment specificity based on presence-absence matrix $A$

- $b$ : a hyperparameter controlling the width of the Laplace prior on $\hat{s}_{m, x, E}(A)$ (i.e., the amount of shrinkage in the estimate)

$-b_{\text {optim }}$ : a value of $b$ optimized for sensitivity and specificity in parametric simulations

$-P\left(e_{x}\right)$ : the prior probability of encountering environment $e_{x}$; we use either an uninformative uniform prior (for bodysites), or take this prior from epidemiological data (for disease comparisons)

- $\widehat{s}_{m, x, E}^{\mathrm{MAP}}(A ; b)$ : a maximum a posteriori (MAP) estimate of environment specificity score for environment $e_{x}$ based on presence-absence matrix $A$ and the shrinkage hyperparameter $b$; in this paper we calculate environmental specificity scores for $x=$ CD (Crohn's disease specificity) and $x=$ Gut (healthy gut specificity) 
- Simulation and censoring analysis

- $\vec{\phi}^{\mathrm{Sim}}$ : a simulated continuous phenotype

- $\vec{I}^{\mathrm{Sim}}$ : a simulated binary genotype (gene presence-absence)

- $\alpha$ : Ives-Garland $\alpha$, the sum of the transition probabilities from 0 to 1 and from 1 to 0 in a Markov model of binary trait evolution across a tree (i.e., a measure of phylogenetic signal in a binary trait)

$-\beta_{0}$ : assigned parameter giving the ancestral state of the simulated genotype $\vec{\phi}^{\mathrm{Sim}}$

$-\beta_{1}$ : assigned parameter giving the degree to which the continuous phenotype $\vec{\phi}^{\mathrm{Sim}}$ affects the binary genotype $\vec{I}^{\mathrm{Sim}}$; a measure of effect size of phenotype on gene

$-\hat{\beta}_{0}^{\text {Prev }}$ : the estimated ancestral state of our prevalence phenotype $\vec{\phi}_{x, E, D}^{\text {Prev }}(A)$, using a Brownian motion model of trait evolution

- $\widehat{\sigma^{2}}$ : the estimated (non-phylogenetic) variance of our prevalence phenotype $\vec{\phi}_{x, E, D}^{\text {Prev }}(A)$

- $\hat{\beta}_{1}^{\text {Test }}$ : estimated value of effect of gene on phenotype from the phylogenetic linear model

- $F$ : ratio of prevalences, comparing taxa with a given gene (numerator) to taxa without (denominator); an alternative measure of effect size of gene on phenotype

- $\hat{\beta}_{1}^{c}$ : when using a bootstrap null, estimated value of effect of null gene on phenotype from the phylogenetic linear model

- $\vec{\phi}^{\mathrm{Unc}^{c}}$ : a version of the phenotype $\vec{\phi}$ where values at the certain limit of detection have been imputed based on a truncated normal

- $\vec{T}^{c}$ : a vector the same length as $\vec{\phi}$ whose elements have been randomly drawn from a truncated normal distribution

- $\hat{\beta}_{1}^{\text {Test }}{ }^{c}$ : an estimated value of effect of gene on the mock-uncensored phenotype $\vec{\phi}^{\mathrm{Unc}^{c}}$

- $K$ : the value at which left-censoring starts for a phenotype $\vec{\phi}$, expressed as standard deviations below the mean

- Compositionality and prevalence accuracy analysis

- $B_{i}$ : an $\|M\| \times\|N\|$ matrix with elements $b_{(m, n)_{i}}$, corresponding to the abundance of microbe $m$ in sample $n$ and simulation $i$

$* B_{i}^{\text {Abs }}$ : absolute abundances

$* B_{i}^{\text {relCN }}$ : relative abundances obtained by column-normalizing $B_{i}^{\mathrm{Abs}}$

* $B_{i}^{\text {Counts: }}$ counts per microbe per sample, obtained by Dirichlet-Multinomial sampling

$* B_{i}^{\text {relDM }}$ : relative abundances obtained by column-normalizing $B_{i}^{\text {Counts }}$

$-c_{m, n}$ : a binary random variable determining whether a microbe $m$ was truly present in a given sample $n$

$-\mu_{m}$ : a microbe-specific average abundance

$-z_{m}$ : a microbe-specific prevalence

- $\theta_{n_{i}}$ : multinomial probability parameters for a given sample $n$ in simulation $i$

$-r_{n}$ : the number of total reads in a sample $n$

- $\alpha_{m}$ : a Dirichlet parameter for a particular microbe $m$ 